\title{
BMJ Open Clinical outcomes and cost-utility of rotator cuff repair surgery by open and arthroscopic techniques: study protocol for a randomised clinical trial
}

\author{
Rafael Pierami (D) , ${ }^{1,2}$ Eliane Antonioli (D) , ${ }^{2}$ Isadora Oliveira, ${ }^{2}$ \\ Isabela Queirós Castro, ${ }^{2}$ Felipe Manente, ${ }^{3}$ Paula Fairbanks, ${ }^{3}$ \\ Eduardo da Frota Carrera, ${ }^{1,2}$ Bruno Akio Matsumura, ${ }^{1,2}$ Mario Lenza ${ }^{2,3}$
}

To cite: Pierami R, Antonioli E, Oliveira I, et al. Clinical outcomes and cost-utility of rotator cuff repair surgery by open and arthroscopic techniques: study protocol for a randomised clinical trial. BMJ Open 2020;10:e043126. doi:10.1136/ bmjopen-2020-043126

- Prepublication history and additional material for this paper are available online. To view these files, please visit the journal online (http://dx.doi. org/10.1136/bmjopen-2020043126).

Received 28 July 2020 Revised 30 November 2020 Accepted 01 December 2020

Check for updates

(c) Author(s) (or their employer(s)) 2020. Re-use permitted under CC BY-NC. No commercial re-use. See rights and permissions. Published by BMJ.

${ }^{1}$ Departamento de Ortopedia, Grupo de Ombro e Cotovelo do Hospital Alvorada Moema, Sao Paulo, Sao Paulo, Brazil ${ }^{2}$ Programa Locomotor, Hospital Israelita Albert Einstein, Sao Paulo, Brazil

${ }^{3}$ Faculdade Israelita de Ciências da Saúde Albert Einstein, Sao Paulo, SP, Brazil

Correspondence to Dr Rafael Pierami; rafael_pierami@hotmail.com

\section{ABSTRACT}

Introduction Rotator cuff injuries account for up to $70 \%$ of pain in the shoulder. However, there remains no consensus on the best surgical treatment for patients with rotator cuff injuries, in terms of the cost-effectiveness and cost-utility of open and arthroscopic methods for rotator cuff repair. The objective of this trial is to compare the efficacy, cost-effectiveness and cost-utility of open and arthroscopic procedures for rotator cuff repair.

Methods and analysis The trial is a two-group, parallel-design, randomised controlled trial. A total of 100 patients with symptomatic rotator cuff lesions will be allocated in either open or arthroscopic technique in a 1:1 ratio, considering smoking (yes or no), lesion size $(\leq 3 \mathrm{~cm}$ or $>3 \mathrm{~cm}$ ) and diabetes (present or absent) as stratification factors. All patients will be included in the same rehabilitation programme after the intervention. The primary outcome measure will be the ConstantMurley Score and the EuroQol-5D-3L score at 48 weeks postsurgery. Secondary outcomes include costeffectiveness, cost-utility, pain, complications and clinical analysis, using the Simple Shoulder Test, Visual Analogue Pain Scale (VAS), integrity of the repair evaluated through $\mathrm{MRI}$, and complications and failures of the proposed methods. For the cost-effectiveness analysis, we will use the VAS and the Constant-Murley Score as measures of effectiveness. For the cost-utility analysis, we will use the EuroQol-5D-3L as a measure of utility in terms of incremental cost per quality-adjusted life-years.

Ethics and dissemination The study has been approved by the local research ethics committee of both institutions: Hospital Israelita Albert Einstein and Hospital Alvorada Moema/Hospital Pró-Cardíaco. The results will be published in a peer-reviewed, open access journal. Trial registration number NCT04146987.

\section{INTRODUCTION}

\section{Background and rationale}

Musculoskeletal injuries are a major cost to the healthcare system. North American data estimate that approximately 4.5 million patients annually seek medical attention due to shoulder pain; of these, 2 million have
Strengths and limitations of this study

- This study is a prospective, randomised trial, which is the best study design to address the research question.

- Methodological analysis is the best option to determine cost-utility and will provide strong evidence.

- It will provide surgeons and healthcare providers with important information about the surgical technique and the cost-effectiveness and cost-utility of the technique

- This study will provide important information about rotator cuff healing and retear rates, which remain unclear in the literature.

- The lack of blinding of patients and surgeons is a limitation of the study design.

some symptoms related to the rotator cuff. About 250000 rotator cuff repair surgeries are performed annually in the USA, and with the continued increase in life expectancy and ageing this number is expected to increase. ${ }^{1-3}$ An evaluation of the primary healthcare system in Cambridge, UK showed that the average frequency of shoulder pain was 9.5 per 1000 individuals. ${ }^{4}$ Of these, $86 \%$ had rotator cuff tendinopathy.

The rotator cuff is a group of four muscles and their tendons that act to stabilise the shoulder and allow for extensive range of motion. Four muscles and their attached tendons make up the rotator cuff: subscapularis, supraspinatus, infraspinatus and teres minor. The long portion of the biceps tendon also contributes to cuff function, which is to stabilise the humeral head in the glenoid cavity, preventing superior migration of the humeral head. ${ }^{5}$

Possible lesions range from tendon degeneration (tendinosis/tendinopathy), through partial tear (articular, interstitial or bursal), 
to complete tear. The aetiology is multifactorial and the main factors associated with tears are tendon degeneration related to ageing, trauma, tendon insertion hypovascularity and genetic factors. ${ }^{6-8}$ Since most lesions are due to wear and degeneration related to ageing, people over 40 years are at greater risk. ${ }^{3}$ Diagnosis is made by associating history and physical examination, along with imaging methods. MRI is considered the method of choice. ${ }^{9-17}$

Treatment of rotator cuff lesion depends on the type of tear, the patient's functional capacity, age and presence of symptoms. In general, tendon degeneration and partial tears are treated non-surgically with physiotherapy, injections and analgesic medications. Complete and incomplete tears that did not respond well to conservative treatment, however, might be treated surgically. ${ }^{32} 18-22$

Among the surgical options, the open method is still considered the gold standard, with good or excellent results in over $90 \%$ of cases. ${ }^{23-25}$ Due to arthroscopy and the evolution of arthroscopic instruments and implants in the last two decades, the arthroscopic repair technique has gained space and is widely used. Some studies ${ }^{23-26}$ did not show superiority of one technique over another in terms of clinical outcomes. On the other hand, since the cost of arthroscopic surgery is supposedly higher due to the required equipment, it is important to establish which option has the best cost-utility ratio. Other published studies suggested that the open method is superior to the arthroscopic method in relation to cost-utility. ${ }^{27-29}$ To date, no study in our country has assessed the cost-utility of the two techniques; considering that the open technique is being left behind, it is important to determine if this technique remains a viable, reliable and cost-effective option for treatment of rotator cuff tears.

\section{Objectives}

Despite the high incidence of rotator cuff tears, there is no consensus on the best method of repair, and neither on which method is the most cost-effective and has the best cost-utility ratio. Therefore, the present study aims to compare the open and arthroscopic methods for rotator cuff repair and determine which has better costeffectiveness ratio.

\section{Trial design}

The trial will be a prospective, randomised controlled clinical trial.

\section{METHODS}

This randomised controlled trial will follow the Consolidated Standards of Reporting Trials statement. ${ }^{30}$ The protocol was developed following the Standard Protocol Items: Recommendations for Interventional Trials guidelines. ${ }^{31}$ It will be performed at Hospital Alvorada Moema (Shoulder and Elbow Surgery Center of Excellence) in São Paulo, Brazil. The cost analysis will be performed by a team from Hospital Israelita Albert Einstein in São
Paulo, Brazil. The project was approved by both hospitals' research ethics committee and registered at ClinicalTrials.gov.

\section{Sample size}

The sample size estimate was obtained to detect differences between the open and arthroscopic repair groups in relation to the primary outcome of the study, ConstantMurley Score (CM) after the intervention. Kukkonen et $a l$ s study ${ }^{32}$ estimated the minimal clinically important difference in CM score at 10.4 points in patients with rotator cuff rupture after 3 months of surgical treatment by the arthroscopic method. The estimated sample size of 45 patients per group, for a total of 90 patients, would reach $90 \%$ power to detect a 10.4 difference between the groups using the CM instrument postoperative score, with an SD of up to 15 points and a significance level of 5\% using Student's t-test. Predicting a loss of around 10\% at 12 months of follow-up, we aim to recruit 50 patients per group (PASS software ${ }^{33}$ ).

\section{Inclusion criteria}

All patients 18 years of age or older presenting with complete rotator cuff tear or a partial rotator cuff tear of at least $50 \%$ of tendon thickness, with symptoms (pain and/or weakness) and where conservative therapy failed, will be included. The tendon tear will be confirmed by MRI.

\section{Exclusion criteria}

Patients with previous shoulder surgery, previous fractures in the affected shoulder, those with limitation in passive range of motion (joint stiffness with an elevation of $90^{\circ}$ or less), radiographic signs of glenohumeral osteoarthritis or neurological injury will be excluded. Patients will also be excluded if they do not wish to participate or are unable to understand or sign the informed consent form (due to conditions such as cognitive impairment or mental illness) or if there are any medical conditions that contraindicate any of the surgical methods.

\section{Randomisation and allocation}

After eligibility assessment, all patients will be informed about the nature and purpose of the study and will only be included after agreeing with the study and signing the informed consent form, which will be obtained by the surgeon who evaluated the patient and indicated the surgery. Patients will be consecutively allocated to one of two proposed treatment methods: open rotator cuff repair or arthroscopic rotator cuff repair. The $\mathrm{R}$ software was used to generate a randomisation list, considering 100 patients to be included in the study and the same probability of allocation for both methods of surgery (open and arthroscopic repair). A stratified randomisation will be performed using the following variables (strata): smoking (yes or no), size of the lesion $(\leq 3 \mathrm{~cm}$ or $>3 \mathrm{~cm}$ ) and diabetes (present or absent). Randomisation will be performed by the REDCap platform (Research Electronic Data Capture; Vanderbilt University, Nashville, 
Tennessee $)^{34} 35$ after the patient has been anaesthetised and prepared for surgery. A person not associated with the study will open the software and acquire one of the two techniques possible and tell the surgeon who will perform the surgery.

\section{Recruitment}

All patients scheduled to be treated by the shoulder surgeons at Hospital Alvorada Moema (Shoulder and Elbow Surgery Center of Excellence) will be enrolled in this trial.

\section{Blinding}

Due to the type of intervention, neither the participants nor the treatment providers can be blinded to treatment allocation. The assessment of primary and secondary outcomes (CM, EuroQol-5D-3L, Visual Analogue Pain Scale (VAS) and Simple Shoulder Test (SST)) and patient-reported outcomes will not be blinded. One of the authors (RP) will assess all other clinical outcomes. The statisticians conducting the analyses will be blinded to treatment status until the analyses have been completed.

\section{Patient and public involvement}

Patients and the public were involved in the design and development of this study. Participation will first occur upon contact between the surgeon and the patient, which is when they will be informed about the study and decide whether or not to participate. At this time, they will be informed about the purpose and importance of the study. During the entire follow-up, patients will be able to enquire with the researchers and surgeons about the project and to make suggestions and complaints about it. All outcome measures will be self-reported. Patients will be assisted by one member of the research team if they have any questions or doubts.

Since the authors agreed to publish the results of this research, patients will not be involved in the dissemination. However, they will be encouraged to disseminate the knowledge in the community.

\section{Intervention methods}

Five surgeons with at least 4 years of surgical technique experience will participate in this study (EFC, MTCA, RP, BAM, VR). Also, shoulder and elbow surgery residents as well as orthopaedics and traumatology residents at Hospital Alvorada Moema and shoulder and elbow surgery residents at Albert Einstein Hospital may participate in the surgery.

\section{Open surgery}

Patients will be positioned in a beach chair position with the affected limb hanging off the edge of the table, allowing manipulation and full range of motion. After standard patient preparation, an anterolateral incision will be made on the shoulder; the deltoid muscle belly will be gently divided along its fibres until exposure of the subdeltoid/subacromial bursa, which will be partially excised to expose the subacromial space and rotator cuff tendons. After mobilisation and release of the ruptured tendons and debridement of the rotator cuff footprint, the tendon repair to the bone will be performed using $5.5 \mathrm{~mm}$ metal anchors ('Super Revo', CONMED, USA), according to the preference and technique chosen by the surgeon. In all cases, the release of the coracoacromial ligament and acromioplasty will be performed.

\section{Arthroscopic technique}

Patients will be positioned in lateral decubitus position, with the limb to be operated attached to a skin traction device and $7 \mathrm{~kg}$ maintaining the shoulder in the following position: abduction of $30^{\circ}-60^{\circ}$ and flexion of $20^{\circ}-30^{\circ}$. After standard patient preparation, a posterolateral incision will be made on the shoulder for optic introduction, with $50 \mathrm{~mm} \mathrm{Hg}$ pressure pump and 0.90 flow, and inspection of the glenohumeral joint. After establishment of all required arthroscopic portals, joint inspection will be performed, and any associated pathologies, if present, will be addressed. With the use of shaver blades, partial bursectomy will be performed and any adherence to the tendon stumps will be released, as well as debridement of the rotator cuff footprint. The tendon will then be reinserted to the bone using metallic $5.5 \mathrm{~mm}$ anchors ('Super Revo', CONMED), according to the preference of each surgeon. The technique used, as well as suture configuration and type of knot used, will be determined by the surgeon according to their preference. After tendon repair, the coracoacromial ligament will be released and acromioplasty will be performed.

\section{Postoperative rehabilitation}

All patients will undergo the same postoperative rehabilitation protocol: use of Velpeau sling for 6 weeks; pendulum exercises starting on the 2nd week; active movement and recovery of the range of motion from the 6th week; and strengthening from the 12th week.

Patients will be oriented to perform home exercises and to be assisted by a physiotherapist twice a week from the sixth week of surgery and on. Approximately 30 sessions of physical therapy will be expected.

\section{Outcome assessment}

Study data will be collected and managed using REDCap (Vanderbilt University) hosted at Hospital Israelita Albert Einstein. ${ }^{34}$ REDCap is a secure, web-based software platform designed to support data capture for research studies, providing (1) an intuitive interface for validated data capture; (2) audit trails for tracking data manipulation and export procedures; (3) automated export procedures for seamless data downloads to common statistical packages; and (4) procedures for data integration and interoperability with external sources.

All study participants will be evaluated preoperatively, at hospital discharge, and at 1, 2, 6, 24 and 48 weeks after the intervention. The CM, VAS, EuroQol5D-3L and SST questionnaires will be filled out by the patient and assessed by the evaluators assigned to the 
intervention. The endpoint of cost-utility analysis will be 48 weeks; clinical outcomes will also be assessed at 6 and 24 weeks.

To prevent loss to follow-up, all patients will be monitored by the REDCap software and alerts will be sent to each patient near time points defined by the investigators: 1 week before every medical consultation and at the 12th week during the rehabilitation process. If the patient fails to fill in any questionnaire or does not attend the medical consultations, the patient will be contacted by phone and email. If a patient becomes not reachable at any time of follow-up, we will consider a lost to follow-up scenario, where in accordance with the intention-to-treat principle appropriate statistical methods for data analysis which consider unbalanced data and loss to follow-up, such as the generalised estimating equation model, will consider all patient observations, even if they fail at some time. Thereby, these patients will not be excluded and all data will be considered.

\section{Primary outcome}

The Brazilian Portuguese version of the $\mathrm{CM}^{36}$ will be measured preoperatively and at 6, 24 and 48 weeks after the intervention. Research assistants (not blinded to the aim of the study) will ask the patients to fill in the CM form validated in Portuguese and measure the range of motion with an analogical goniometer. The $\mathrm{CM}$ scale covers different domains of shoulder function (pain, activities of daily living, range of motion and power), scoring each of them. The score ranges from 0 to 100 , with higher scores indicating better function. ${ }^{36} \mathrm{CM}$ is one of the most commonly used scales to score shoulder function and is considered the gold standard in Europe. ${ }^{37} 38$ It is reliable in the detection of shoulder improvement after surgical procedures, and its strong correlation with shoulder-specific diseases, especially rotator cuff, and its reliability make it a good scoring system for clinical research.

The EuroQol-5D-3L (European Quality of Life), a generic score developed to describe health-related quality of life, ${ }^{30}$ will also be assessed preoperatively and at 6,24 and 48 weeks postoperatively. This score includes five health domains: mobility, self-care, usual activities, pain/discomfort and anxiety/depression. Each domain has three levels: no problem, some problems and extreme problems. In addition, the EuroQol-5D-3L has a visual analogue scale where the participant assigns a value between 0 and 100 to his or her own health condition, where 100 means 'the best imaginable health status' or 'the best health state you can imagine' and 0 means 'the worst imaginable health state' or 'the worst health state you can imagine'. This is used to obtain a respondent's stated preference values, not to record their own health state. ${ }^{39}$ At the end of its application, the EuroQol5D-3L will provide a unique numerical value that can be used for longitudinal comparison between different time periods.

\section{Secondary outcomes}

Clinical outcomes will also be assessed by SST, validated in Portuguese, ${ }^{40}$ preoperatively and at 6, 24 and 48 weeks after the procedure. SST is a simple, quick and widely used questionnaire for shoulder function measurement; it consists of 12 dichotomous questions answered by the patients themselves. Each positive answer (yes) is given a score. At the end of the questionnaire the percentage of positive answers (score) is obtained, and the higher the percentage the better the shoulder function. Other outcomes measured will be VAS at hospital discharge and at 1, 2, 6, 24 and 48 weeks after the intervention. This scale allows pain intensity to be measured with maximum interobserver reproducibility. It consists of a $10 \mathrm{~cm}$ straight line, with the ends determining the limits of pain sensation (no pain; worst pain ever experienced); the distance between zero (no pain) and the patient's demarcation defines the intensity of pain. ${ }^{41}$

Complications and failures of the proposed methods will also be assessed. Failures will be characterised as the need for additional surgical procedures and/or change of the initially proposed procedure. Patients who, for any reason, demonstrate treatment failure or require additional interventions will be followed up and their results included in the group in which they were initially randomised, according to the intention-to-treat principle.

At the final follow-up (48 weeks), the integrity and healing of repaired rotator cuff will be assessed by MRI.

\section{Cost-effectiveness}

Cost-effectiveness and cost-utility analyses will be assessed by estimating the direct and indirect costs to the private healthcare system at 48 weeks. The perspective adopted in the study will be social costs, the direct and indirect medical costs. The set timeframe will be 48 weeks and a sensitivity analysis will be performed with the cost data, considering $0 \%-5 \%$ discount rate to define the optimal discount rate for the data, according to the methodological guidelines for economic evaluation of health technologies by the Brazilian Ministry of Health. ${ }^{42-44}$ Direct medical costs include hospitalisation costs, costs related to arthroscopic instruments (eg, cannulas, shaver blades, suture passer, ablator), medical fees and medication costs. Indirect costs include costs of absence from work, which will be estimated by the patient-reported number of days away from work multiplied by the average wage rate of the current year. The costs will be converted from Brazilian reais to US dollars and brought to the cost schedule of the current year to avoid the inflation having an effect on the analysis of medical inputs. For the cost-effectiveness analysis, the VAS and the CM will be used as measures of effectiveness. For the cost-utility analysis, the EuroQol$5 \mathrm{D}-3 \mathrm{~L}$ will be used as a measure of utility. The timetable of outcomes assessment is described in table 1 .

\section{Data analysis}

Descriptive analyses of variables will be based on absolute frequencies and percentages for categorical variables, 
Table 1 Timetable of assessment

Study period

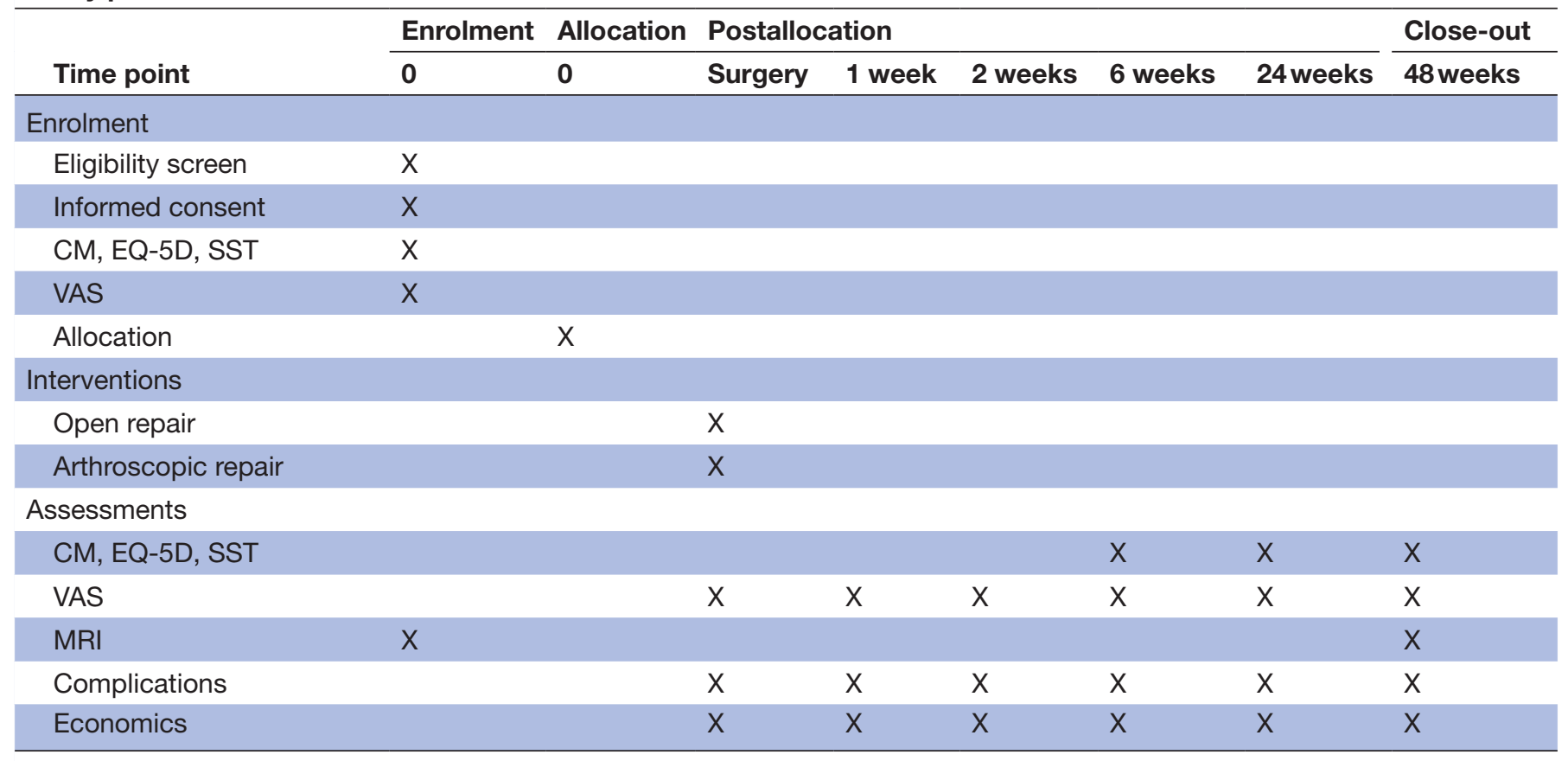

CM, Constant-Murley Score; EQ-5D, EuroQOL-5D-3L; SST, Simple Shoulder Test; VAS, Visual Analogue Pain Scale.

and summary measures as mean and SD or median and quartiles, as well as minimum and maximum values for numerical variables. ${ }^{45}$ Clinical scores will be represented by individual profile graphs separately by the surgical technique group.

The groups will be compared according to the presence of categorical clinical outcomes (failures, complications and healing integrity) by $\chi^{2}$ or Fisher's exact tests, depending on the distribution observed after data collection.

For inferential analysis of continuous variables of clinical outcomes, mixed models will be used, and if the normal distribution is not adequate generalised mixed models will be used. ${ }^{46}$ The models will have time effects (preoperative and 6, 24 and 48 weeks after intervention), surgical technique group (open repair or arthroscopic repair) and the interaction effect between time and group. The size of the lesion $(\leq 3 \mathrm{~cm}$ or $>3 \mathrm{~cm})$ will also be included in the models as a control variable, seeking to avoid possible biases.

Analyses will be performed using the SPSS V.24.0 program ${ }^{47}$ considering a significance level of $5 \%$.

\section{Safety}

There will be no benefit to participants beyond what is expected from correcting the rotator cuff tear, expecting an improvement in pain and function of the affected shoulder. The risks of the present study are those inherent in any surgical treatment and anaesthetic procedure, such as surgical wound infection, scar formation, pain, decrease in shoulder range of motion, rotator cuff tear and neurovascular injury. If any complications occur, all patients will be treated by the same surgical team until the complication is healed.

Both surgical techniques have the same goal, that is, to repair the ruptured tendon to the bone. The open technique requires a larger incision, as well as greater surgical dissection and manipulation of the deltoid muscle, which may cause greater postoperative pain and weakness of this muscle, in addition to causing a slightly larger scar. However, it provides great visualisation and manipulation and mobilisation capability of the ruptured tendon, providing a safer and tension-free repair.

The arthroscopic technique is performed with some point-shaped cuts in the shoulder, usually three or four. Due to the smaller incisions, it requires less muscle manipulation, which theoretically would cause less postoperative pain and less muscle weakness of the deltoid muscle. It also results in minor scars. However, this technique requires more surgeon experience and the mobilisation of the ruptured tendon(s) is limited. Using a large amount of saline may cause oedema on the operated shoulder, which usually resolves after the first 12 hours of surgery.

Finally, there is minimal risk of loss of data confidentiality; all data will be managed, stored and protected by REDCap software. ${ }^{345}$ Only the main investigator will have access to all the data during the entire trial period. Any adverse event will be reported to the researchers involved and communicated to the main investigator according to the institutional review boards description. 


\section{Ethics and dissemination}

The study has been approved by the local research ethics committee from both institutions: Hospital Israelita Albert Einstein (CAAE 19182619.3.1001.0071) and Hospital Alvorada Moema/Hospital Pró-Cardíaco (CAAE 19182619.3.2002.5533). Digital, informed consent (online supplemental material) to participate will be obtained from all participants through the REDCap software. ${ }^{3435}$

This study was developed and will follow the International Conference Guideline for Good Clinical Practice to ensure that the data and the results are credible and that the rights, integrity and confidentiality of the trial subjects are protected and respected. ${ }^{48} 49$

All authors agreed to publish the results of the present study in a peer-reviewed, open access journal, regardless of the results and conclusions found. All data will be available on request.

\section{DISCUSSION}

There is no consensus on which surgical treatment for patients with degenerative rotator cuff injuries is the most cost-effective. Several studies ${ }^{27285051}$ suggest that the open repair method is more cost-effective than the arthroscopic method and results in the same clinical outcome at a lower cost. Adla et $a l^{27}$ in a prospective, non-randomised study showed that both techniques lead to the same clinical outcomes. The cost of arthroscopic surgery was higher than open surgery, mainly due to the cost of the suture anchor, which was used only in the arthroscopic group. It is important to note that, in most of the open surgeries, repair was performed through transosseous sutures. Köse $e t a l^{28}$ in a retrospective study also demonstrated similar clinical outcomes, although the cost of arthroscopic procedure was much higher. Importantly, the open repair technique was performed using transosseous sutures and the arthroscopic method using suture anchors. Also, the open repair group required longer stay at the hospital. Hui $e$ et $a \tilde{l}^{0}$ in a retrospective cohort study described a significantly higher cost for the arthroscopic procedure, compared with the open repair, evaluating only the in-hospital costs, but with the same clinical outcomes. However, it is important to emphasise that open repair was performed using transosseous sutures, without suture anchors, and that the arthroscopic group needed a longer surgery time. Churchill and Ghorai ${ }^{51}$ using the New York Ambulatory Database System, with a total of 5224 cuff repair surgeries, of which 1334 were open repair and 3890 were arthroscopic repair, showed that the mini-open rotator cuff repair costs significantly less than the arthroscopic repair and requires significantly less surgical time. However, no clinical outcomes have been analysed in this study, making it impossible to determine the cost-effectiveness ratio. An important study by Carr et $a \tilde{l}^{2}$ carried out as a prospective multicentre randomised clinical trial concluded that there is no difference in the effectiveness and cost-effectiveness between open repair surgery and arthroscopic surgery after 24 months of follow-up, even with the higher initial costs with arthroscopy surgery. An economic evaluation of the data from this study was carried out showing that the incremental cost-effectiveness was uncertain and that arthroscopic repair surgery was slightly more costly and less effective than open repair surgery.

Thus, despite the high incidence of rotator cuff tear, there is insufficient evidence to determine the best method for treating these injuries. The present study proposes to answer the clinical question of which method, open or arthroscopic, has the best cost-utility in the surgical treatment of rotator cuff tear, providing conclusive, good-quality evidence for and contributing to the evidence base of methods used to treat rotator cuff injuries.

\section{Trial status}

- Protocol trial version: 5; date: 11 December 2020.

- Recruitment start date: August 2020.

- Recruitment estimated end date: December 2021.

- Recruiting.

\section{Registry}

The project is registered at the ClinicalTrials.gov database (https:// clinicaltrials.gov/ct2/show/NCT04146987? term $=$ NCT04146987\&draw $=2 \&$ rank $=1$ ).

\section{Dissemination policy}

All the authors are committed and agree to publish the full results of the research regardless of the final results.

\section{Data monitoring committee}

Since this trial has a short duration and both surgical techniques have known minimal risks, there is no need for a data monitoring committee.

Collaborators Vitor Rodrigues Domingues, Maria Thereza Calil Angelini, Rubens Jose Pierami Neto.

Contributors All listed authors had substantial contribution to the conception and development of this study, revised and approved the final version, and are accountable for all aspects of the study. The authors ensure that all questions related to the accuracy or integrity of any part of the project are appropriately investigated and resolved. ML is the chief investigator; he conceived the study and led the proposal and protocol development. RP is the lead trial methodologist and helped in conceiving the study and its development. EA contributed to study design and development of the proposal. 10 contributed to study design related to qualityadjusted life-years. IQC is responsible for cost analysis. FM and PF helped in the English translation and registration/publication of the trial. EFC and BAM helped in conceiving the study and its development.

Funding This study is supported by Fundação de Amparo à Pesquisa do Estado de São Paulo (FAPESP 2019/02159-3).

Competing interests None declared.

Patient consent for publication Obtained.

Provenance and peer review Not commissioned; externally peer reviewed.

Supplemental material This content has been supplied by the author(s). It has not been vetted by BMJ Publishing Group Limited (BMJ) and may not have been peer-reviewed. Any opinions or recommendations discussed are solely those of the author(s) and are not endorsed by BMJ. BMJ disclaims all liability and responsibility arising from any reliance placed on the content. Where the content includes any translated material, BMJ does not warrant the accuracy and reliability of the translations (including but not limited to local regulations, clinical guidelines, 
terminology, drug names and drug dosages), and is not responsible for any error and/or omissions arising from translation and adaptation or otherwise.

Open access This is an open access article distributed in accordance with the Creative Commons Attribution Non Commercial (CC BY-NC 4.0) license, which permits others to distribute, remix, adapt, build upon this work non-commercially, and license their derivative works on different terms, provided the original work is properly cited, appropriate credit is given, any changes made indicated, and the use is non-commercial. See: http://creativecommons.org/licenses/by-nc/4.0/.

ORCID iDs

Rafael Pierami http://orcid.org/0000-0002-1745-4362

Eliane Antonioli http://orcid.org/0000-0002-8563-877X

\section{REFERENCES}

1 lii RCMet al. The societal and economic value of rotator cuff repair 2013:1993-2000.

2 Kuye IO, Jain NB, Warner L, et al. Economic evaluations in shoulder pathologies: a systematic review of the literature. J Shoulder Elbow Surg 2012;21:367-75

3 American Academy of Orthopaedic Surgeons. Management of rotator cuff injuries clinical practice guideline. Orthoguidelines 2019.

4 Ostör AJK, Richards CA, Prevost AT, et al. Diagnosis and relation to general health of shoulder disorders presenting to primary care. Rheumatology 2005;44:800-5.

5 Favard L, Bacle G, Berhouet J. Rotator cuff repair. Joint Bone Spine 2007;74:551-7.

6 pp. Gumina S, Carbone S, Campagna V, et al. The impact of aging on rotator cuff tear size. Musculoskelet Surg 2013;97 Suppl 1:69-72.

7 Nové-Josserand L, Walch G, Adeleine P, et al. [Effect of age on the natural history of the shoulder: a clinical and radiological study in the elderly]. Rev Chir Orthop Reparatrice Appar Mot 2005;91:508-14.

8 Yamaguchi K, Tetro AM, Blam O, et al. Natural history of asymptomatic rotator cuff tears: a longitudinal analysis of asymptomatic tears detected sonographically. J Shoulder Elbow Surg 2001;10:199-203.

9 Tempelaere C, Pierrart J, Lefèvre-Colau M-M, et al. Dynamic threedimensional shoulder MRI during active motion for investigation of rotator cuff diseases. PLoS One 2016;11:e0158563.

10 Sela Y, Eshed I, Shapira S, et al. Rotator cuff tears: correlation between geometric tear patterns on MRI and arthroscopy and preand postoperative clinical findings. Acta Radiol 2015;56:182-9.

11 Teefey SA, Rubin DA, Middleton WD, et al. Detection and quantification of rotator cuff tears. Comparison of ultrasonographic, magnetic resonance imaging, and arthroscopic findings in seventyone consecutive cases. J Bone Joint Surg Am 2004;86:708-16.

12 Jason E AOG, Hsu Steven B, Lippitt FA. Matsen III, "Rockwood and Matsen's The Shoulder, 5th Edition: The Rotator Cuff," in Rockwood and Matsen's The Shoulder. 5th edn. Elsevier, 2016: 651-719.

13 Yamakawa S, Hashizume H, Ichikawa N, et al. Comparative studies of MRI and operative findings in rotator cuff tear. Acta Med Okayama 2001:55:261-8.

14 Roy J-S, Braën C, Leblond J, et al. Diagnostic accuracy of ultrasonography, MRI and Mr arthrography in the characterisation of rotator cuff disorders: a systematic review and meta-analysis. $\mathrm{Br} \mathrm{J}$ Sports Med 2015;49:1316-28.

15 Lenza M, Buchbinder R, Takwoingi Y, et al. Magnetic resonance imaging, magnetic resonance arthrography and ultrasonography for assessing rotator cuff tears in people with shoulder pain for whom surgery is being considered. Cochrane Database Syst Rev 2013:CD009020.

16 Handoll HH, Hanchard NC, Lenza M, et al. Rotator cuff tears and shoulder impingement: a tale of two diagnostic test accuracy reviews. Cochrane Database Syst Rev 2013;10:ED000068.

17 Hanchard NCA, Lenza M, Handoll HHG, et al. Physical tests for shoulder impingements and local lesions of bursa, tendon or labrum that may accompany impingement. Cochrane Database Syst Rev 2013:CD007427.

18 Seida JC, LeBlanc C, Schouten JR, et al. Systematic review: nonoperative and operative treatments for rotator cuff tears. Ann Intern Med 2010;153:246.

19 Ainsworth R, Lewis JS. Exercise therapy for the conservative management of full thickness tears of the rotator cuff: a systematic review. Br J Sports Med 2007;41:200-10.

20 Eljabu W, Klinger HM, von Knoch M. The natural history of rotator cuff tears: a systematic review. Arch Orthop Trauma Surg 2015;135:1055-61.
21 Oliva F, Piccirilli E, Bossa M, et al. I.S.Mu.L.T - Rotator Cuff Tears Guidelines. Muscles Ligaments Tendons J 2015;5:227-63.

22 Arce G, Bak K, Bain G, et al. Management of disorders of the rotator cuff: proceedings of the ISAKOS upper extremity Committee consensus meeting. Arthroscopy 2013;29:1840-50.

23 van der Zwaal P, Thomassen BJW, Nieuwenhuijse MJ, et al. Clinical outcome in all-arthroscopic versus mini-open rotator cuff repair in small to medium-sized tears: a randomized controlled trial in 100 patients with 1-year follow-up. Arthroscopy 2013;29:266-73.

24 Morse K, Davis AD, Afra R, et al. Arthroscopic versus mini-open rotator cuff repair: a comprehensive review and meta-analysis. Am J Sports Med 2008;36:1824-8.

$25 \mathrm{Ji} \mathrm{X}, \mathrm{Bi} \mathrm{C}$, Wang F, et al. Arthroscopic versus mini-open rotator cuff repair: an up-to-date meta-analysis of randomized controlled trials. Arthroscopy 2015;31:118-24.

26 Huang R, Wang S, Wang Y, et al. Systematic review of AllArthroscopic versus Mini-Open repair of rotator cuff tears: a metaanalysis. Sci Rep 2016;6:22857.

27 Adla DN, Rowsell M, Pandey R. Cost-Effectiveness of open versus arthroscopic rotator cuff repair. J Shoulder Elbow Surg 2010;19:258-61.

28 Köse KC, Tezen E, Cebesoy O, et al. Mini-open versus allarthroscopic rotator cuff repair: comparison of the operative costs and the clinical outcomes. Adv Ther 2008;25:249-59.

29 Vitale MA, Vitale MG, Zivin JG, et al. Rotator cuff repair: an analysis of utility scores and cost-effectiveness. J Shoulder Elbow Surg 2007;16:181-7.

30 Sanders GDet al. Recommendations for conduct, methodologica practices, and reporting of cost-effectiveness analyses: second panel on cost-effectiveness in health and medicine. JAMA 2016.

31 Chan A-W, Tetzlaff JM, Altman DG, et al. Spirit 2013 statement: defining standard protocol items for clinical trials. Ann Intern Med 2013;158:200.

32 Kukkonen J, Kauko T, Vahlberg T, et al. Investigating minimal clinically important difference for constant score in patients undergoing rotator cuff surgery. J Shoulder Elbow Surg 2013;22:1650-5.

33 L NCSS. Pass 14 power analysis and sample size software. Kaysville, Utah, USA, 2015

34 Harris PA, Taylor R, Thielke R, et al. Research electronic data capture (REDCap)--a metadata-driven methodology and workflow process for providing translational research informatics support. J Biomed Inform 2009;42:377-81.

35 Harris PA, Taylor R, Minor BL, et al. The REDCap Consortium: building an international community of software platform partners. $J$ Biomed Inform 2019;95:103208.

36 Barreto RPG, Barbosa MLL, Balbinotti MAA, et al. Versão brasileira do Constant-Murley score (CMS-BR): validade convergente $E$ de constructo, consistência interna E unidimensionalidade. Revista Brasileira de Ortopedia 2016;51:515-20.

37 Angst F, Schwyzer H-K, Aeschlimann A, et al. Measures of adult shoulder function: disabilities of the arm, shoulder, and hand questionnaire (DASH) and its short version (QuickDASH), shoulder pain and disability index (SPADI), American shoulder and elbow surgeons (ases) Society standardized shoulder. Arthritis Care Res 2011;63:S174-88.

38 Constant CR, Gerber C, Emery RJH, et al. A review of the constant score: modifications and guidelines for its use. J Shoulder Elbow Surg 2008;17:355-61.

39 Noronha F. Associação Portuguesa de Economia dA Saúde Lara de Noronha E Ferreira 2002;46.

40 Neto JOB, Gesser RL, Steglich V, et al. Validation of the simple shoulder test in a Portuguese-Brazilian population. is the latent variable structure and validation of the simple shoulder test stable across cultures? PLoS One 2013;8:e62890-8.

41 Delgado DA, Lambert BS, Boutris N, et al. Validation of digital visual analog scale pain scoring with a traditional Paper-based visual analog scale in adults. J Am Acad Orthop Surg Glob Res Rev 2018;2:e088.

42 Ribeiro RAet al. Diretriz metodológica para estudos de avaliação econômica de tecnologias em saúde no Brasil methodological guidelines for economic evaluation studies of health technologies in Brazil. J Bras Econ Saúde 2016.

43 Ribeiro RAet al. Methodological guidelines for economic evaluation studies of health technologies in Brazil. J Bras Econ Saúde 2016.

44 Siegel JE. Recommendations for reporting cost-effectiveness analyses. panel on cost-effectiveness in health and medicine. JAMA J Am Med Assoc 1996;276:1339-41.

45 Ludbrook J. Practical statistics for medical research. Australian and New Zealand Journal of Surgery 1991. 
46 Faraway JJ. Extending the linear model with R: generalized linear, mixed effects and nonparametric regression models 2006.

47 BM Corp. Ibm SPSS statistics for windows, version 24.0 2016;2016.

48 Vijayananthan A, Nawawi O. The importance of good clinical practice guidelines and its role in clinical trials. Biomed Imaging Interv $J$ 2008;4:e5.

49 Englev E, Petersen KP. ICH-GCP guideline: quality assurance of clinical trials status and perspectives. Ugeskr. Laeger, 2003.

50 Hui YJ, Teo AQA, Sharma S, et al. Immediate costs of mini-open versus arthroscopic rotator cuff repair in an Asian population. $J$ Orthop Surg 2017;25:230949901668449-6.
51 Churchill RS, Ghorai JK. Total cost and operating room time comparison of rotator cuff repair techniques at low, intermediate, and high volume centers: mini-open versus all-arthroscopic. J Shoulder Elbow Surg 2010;19:716-21.

52 Carr AJ, Cooper CD, Campbell MK, et al. Clinical effectiveness and cost-effectiveness of open and arthroscopic rotator cuff repair [the UK Rotator Cuff Surgery (UKUFF) randomised trial]. Health Technol Assess 2015;19:1-218. 